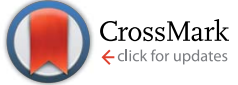

Cite this: RSC Adv., 2017, 7, 14688

\title{
One-step preparation of conjugated homopolymer sub-microspheres via a controllable supramolecular approach toward optoelectronic applications $\dagger$
}

\author{
Mengna Yu,,$^{+a}$ Bin Liu, $t^{a}$ Jinyi Lin, ${ }^{* b c}$ Lubing Bai, ${ }^{b}$ Haifeng Ling, ${ }^{a}$ Wensai Zhu, ${ }^{b}$ \\ Linghai Xie, ${ }^{\text {*a }}$ Mingdong $\mathrm{Yi}^{\mathrm{a}}{ }^{\mathrm{a}}$ Jiangpu Wang ${ }^{\mathrm{b}}$ and Wei Huang ${ }^{\star a b}$
}

Received 30th December 2016 Accepted 23rd February 2017

DOI: 10.1039/c6ra28859f

rsc.li/rsc-advances

\begin{abstract}
Supramolecular poly\{9-[4-(octyloxy)phenyl]fluoren-9-ol-2,7-diyl\} (PPFOH)-based conjugated polymer sub-microspheres (CPSMs) with diameters ranging from $150 \mathrm{~nm}$ to several micrometres are constructed via "one step' solvent evaporation induced interface self-assembly (SEIS). Their diameter and packing mode can be optimized by controlling the preparation parameters, which pave the way to construct colloidal crystal and light-emitting diodes.
\end{abstract}

In the last several decades, conjugated polymers (CPs) have been widely applied in optoelectronic devices, such as polymer light-emitting diodes (PLEDs), polymer solar cells (PSC), polymer lasers and sensors, because of their mechanical flexibility, and low-cost manufacturing as well as their versatile chemical design and synthesis..$^{1-3}$ Compared to the $\mathrm{p}-\mathrm{n}$ (also called donor-acceptor) molecular engineering strategy for constructing optoelectronic materials, ${ }^{4,5}$ the micro/nano-approach can act as an alternative with a higher degree of freedom. For example, it not only controls the energy transfer, charge separation and photophysical characteristics of CPs by manipulating molecular self-assembly behaviour, but also simplifies the fabrication processes of flexible optoelectronic devices. ${ }^{6,7}$ Among various CP-based multi-dimensional micro/ nanostructures, conjugated polymer sub-microspheres (CPSMs) can be used as microscopic building blocks to fabricate organic optoelectronic devices by high-throughput ecofriendly solution processing, ${ }^{8,9}$ particularly the self-assembly of three dimensional (3D) photonic crystals. ${ }^{10,11}$ Other than the sub-microspheres prepared from conventional non-

\footnotetext{
${ }^{a}$ Center for Molecular Systems and Organic Devices (CMSOD), Key Laboratory for Organic Electronics and Information Displays, Institute of Advanced Materials (IAM), Jiangsu National Synergetic Innovation Center for Advanced Materials (SICAM), Nanjing University of Posts \& Telecommunications, 9 Wenyuan Road, Nanjing 210023, China. E-mail: iamlhxie@njupt.edu.cn

${ }^{b}$ Key Laboratory of Flexible Electronics (KLOFE), Institute of Advanced Materials (IAM), Jiangsu National Synergetic Innovation Center for Advanced Materials (SICAM), Nanjing Tech University (NanjingTech), 30 South Puzhu Road, Nanjing 211816, China.E-mail:wei-huang@njtech.edu.cn; iamjylin@njtech.edu.cn

${ }^{\circ}$ Department of Physics and Centre for Plastic Electronics, The Blackett Laboratory, Imperial College London, Prince Consort Road, London SW7 2AZ, UK

$\dagger$ Electronic supplementary information (ESI) available. See DOI: $10.1039 / \mathrm{c} 6 \mathrm{ra} 28859 \mathrm{f}$

\$ Mengna Yu and Bin Liu contributed equally.
}

conjugated or doped polymers, ${ }^{12-14}$ CPSMs-based photonic crystals are expected to show enhanced electroluminescence (EL), no-threshold laser oscillation, and highly efficient photoelectric conversion, ${ }^{15-18}$ such as long-lived excitons, and light confinement. ${ }^{10,11,19,20}$ Thus, it is significant to develop one class of CPs system, as well as a facile method to construct pure CPSMs.

Up to date, the majority of reported polymer micro/ nanoparticles was constructed by direct polymerization techniques, such as dispersion and miniemulsion polymerization, ${ }^{3,6,21}$ or post-polymerization method (multi-steps miniemulsification and precipitation). ${ }^{10,11,22}$ In order to fabricate conjugated polymer micro/nanoparticle's active films or mesoscopic structure, it need multi-steps purification to remove stabilizer for mini-emulsification (diameters ranging from $100 \mathrm{~nm}$ to several micrometres) and/or slowly dry a suspension to distribute/pack particles on precleaned substrates for the precipitation method (sizes ranging from several nanometres to hundreds nanometres) attributed to the lower particles concentration in precursor suspension. ${ }^{22,23}$ What's more, the critical matter is that the synergistic effect of rigidity and hydrophobicity of the aromatic conjugated backbone structure makes it difficult to give isotropic spherical assemblies by postpolymerization, which is out of question for the non-conjugated polymers like polystyrene (PS) and poly(methyl methacrylate) (PMMA). ${ }^{24,25}$ As a result of high planarity, rigidity and hydrophobicity, CPs chains tend to pack together and self-assemble into anisotropic nano-aggregates by post-polymerization method, ultimately generate irregular zero-dimensional (OD) nanoparticles or dots, ${ }^{22,25}$ one-dimensional (1D) fibril, ${ }^{7}$ even two-dimensional (2D) layered structures, ${ }^{26}$ but structurally isotropic spheres are rarely reported. ${ }^{27,28}$ Recently, alternating copolymers with two different $\pi$-conjugated components were 
demonstrated to form well-shaped CPSMs quantitatively with diameters ranging from a few hundreds of nanometres to several micrometres, ascribed to large steric hindrance on their backbone that reduces planarity of the polymers and inhibits anisotropic packed growth. ${ }^{10}$ However, up to now, it is rarely post-polymerization method to construct well-defined, widely size-tunable spherical sub-microstructures prepared from $\pi$ conjugated homopolymer toward potential optoelectronic application, especial colloidal crystal.

Supramolecular functionalization imparts $\pi$-conjugated homopolymer with abundant self-assembly behaviours to construct multi-dimensional superstructures. ${ }^{29,30}$ Accordingly, supramolecular strategy not only endows CPs with selfassembly ability, but also reserves their optoelectronic properties when taking into consideration of the practical applications. ${ }^{31,32}$ Over fast precipitation and evaporation methods, supramolecular approach provides a flexible driving force to induce the formation of CPSMs, which can be attributed to the thermodynamically favourable lowest surface per volume, similar to amphiphilic homopolymers and block copolymers. Herein, we explore the preparation of spherical assemblies of supramolecular conjugated polymer $(\mathrm{PPFOH}),{ }^{33}$ modified with the hydroxyl groups, which are generated by supramolecular interactions during the process of "one step" solvent evaporation induced interface self-assembly (SEIS) (Scheme 1). In our previous work, we constructed PPFOH-based small irregular nanoparticles with the size tuning from $200 \mathrm{~nm}$ to $40 \mathrm{~nm}$ via reprecipitation method. ${ }^{31}$ Here, amphiphilicity of $\mathrm{PPFOH}$ in the $\mathrm{N}, \mathrm{N}$-dimethylformamide (DMF, high boiling point of $153{ }^{\circ} \mathrm{C}$ ) makes polymer chains self-assemble into isotropic CPSMs with the lowest surface energy during slow evaporation without daylight. PPFOH CPSMs tend to show well-shaped CPSMs quantitatively with diameters ranging from a few hundreds of nanometres to several micrometres, as well as a size-dependent emission property. Tentatively, preliminary explorations of PPFOH-based CPSMs toward potential application in photonic crystal and PLEDs are also described here.

As demonstrated in our previous work, PPFO8 (without hydroxyl) exhibited extremely poor solubility in DMF for the large difference of solubility parameters between PPFO8 $(\sim 9.5$ $\left.\left(\mathrm{cal} \mathrm{cm}^{-3}\right)^{1 / 2}\right)$ and DMF (12.1 $\left.\left(\mathrm{cal} \mathrm{cm}^{-3}\right)^{1 / 2}\right)$, indicated DMF is an immiscible solvent for non-polar polyfluorene backbone structures (Scheme 1)..$^{33}$ On the contrary, PPFOH showed good solubility in DMF for the formation of hydrogen bond between hydroxyl and carbonyl of DMF. In order to further confirm this assumption, the ${ }^{1} \mathrm{H}$ NMR spectra of TFOHC8 (trimer of 9-ol-

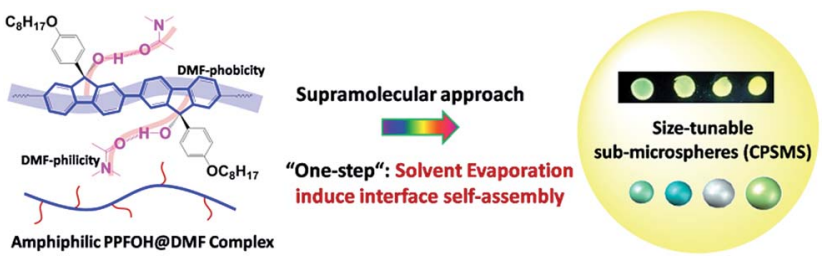

Scheme 1 Illustrate model of PPFOH-based CPSMs via solvent evaporation induced interface self-assembly (SEIS) method. fluorene) in $\mathrm{CDCl}_{3}$ (Fig. $\mathrm{S} 1 \dagger$ ) were investigated, where the TFOHC8 acted as a model of $\mathrm{PPFOH}$ to directly examine whether the hydrogen bonds formation or not between DMF and PPFOH. Firstly, when only TFOHC8 was dissolved in $\mathrm{CDCl}_{3}$, an apparent signal at $\delta(2.495 \mathrm{ppm})$ can be observed, which was attributed to the $\mathrm{H}$ atom of hydroxyl at 9 position of the fluorene unit. With the addition of a small amount of DMF, the aromatic signals downshifted a little, but the $\delta$ of $\mathrm{H}$ atom in hydroxyl upshifted from 2.495 to $4.276 \mathrm{ppm}$, which suggested the formation of a strong interaction between $\mathrm{DMF}(\mathrm{C}=\mathrm{O})$ and hydroxyl $(-\mathrm{OH})$. Meanwhile, the $\delta$ of $\mathrm{H}$ atom at b site of DMF shifted from 7.955 to $7.896 \mathrm{ppm}$, which further confirmed the formation of hydrogen bonds. On this basis, we propose such a model that hydroxyl groups prefer to interact with DMF molecules (DMF-philicity, similar to hydrophilicity) but DMF molecules are immiscible units with the backbones of polyfluorene (DMF-phobicity, hydrophobicity), which makes PPFOH@DMF complex show a slightly amphiphilic property in DMF solution (Scheme 1). Consequently, similar to the spherical self-assembly of the amphiphilic homopolymers and block copolymers, ${ }^{29}$ amphiphilic PPFOH@DMF complexes tend to self-assemble into spherical nanostructures by the control of external conditions.

Subsequently, we attempted to prepare the regular nanostructures of $\mathrm{PPFOH}$ by making use of the amphiphilic property in DMF solution. Reasonably, CPSMs can be obtained from DMF solution of PPFOH on a solid substrate (such as silicon wafer, Fig. 1) via solvent evaporation processing. It is worth noting that conjugated polymers akin to $\mathrm{PPFOH}$ modified with hydroxyl groups also show excellent self-assembly behaviour to construct high quality CPSMs (Fig. S2 $\dagger$ ), which preliminary demonstrated the universality of amphiphilic CPs in terms of spherical self-assembly. Furthermore, the diameters of CPSMs increased gradually with the increasing initial concentrations of DMF solutions (Fig. 1e). As shown in Fig. 1a-d, the average diameters of CPSMs are approximately 150-200 $\mathrm{nm}$ for $1 \mathrm{mg}$ $\mathrm{mL}^{-1}, 400 \mathrm{~nm}$ for $10 \mathrm{mg} \mathrm{mL} \mathrm{m}^{-1}, 700 \mathrm{~nm}$ for $30 \mathrm{mg} \mathrm{mL}^{-1}$, and $2000 \mathrm{~nm}$ for $50 \mathrm{mg} \mathrm{mL} \mathrm{m}^{-1}$, respectively. The increased sizes of CPSMs are likely derived from the high density of the PPFOH chains in the higher concentration. Meanwhile, PPFOH-based CPSMs can also be generated on other substrates, such as glass, quartz plate as well as $\mathrm{Cu}$ grid covered with carbon membrane (Fig. S3†), which demonstrated that "one step" SEIS method in terms of preparing CPSMs thin-film can be implemented on various kinds of substrates or electrodes. Moreover, it is easy to find that CPSMs show more uniform spherical shapes on the interface with the lowest surface energy, because of the effective molecular rearrangement during the DMF molecules evaporation process. In addition, PPFOH-based CPSMs were revealed to be solid by transmission electron microscope (TEM) analysis (Fig. S4 $\dagger$ ). As shown in Fig. S5, $\dagger$ a larger, stronger and continuous ring diffraction in GIXD pattern suggested the isotropic amorphous states in CPSMs, which also supported the assumption of isotropic property of CPSMs. In order to further demonstrate the validity of amphiphilic property of $\mathrm{PPFOH}$, we tried dimethyl sulfoxide (DMSO) and dimethylacetamide (DMAc) solutions as well, where the 
(a)

hydrophilicity can be induced by the formation of hydrogen bonds between solvent and hydroxyl of PPFOH, and induced polymer chains self-assemble into CPSMs (Fig. S6†). By contrary, continuous PPFOH films instead of CPSMs were obtained in other solutions, such as chloroform, tetrahydrofuran and toluene solutions (Fig. S7†), which further indicated that amphiphilic property of PPFOH in DMF solution is the driving force for PPFOH chains self-assembling into CPSMs films.

Not surprising, no CPSMs were formed for the corresponding PPFO8 solution at the same condition for the poor solubility in DMF. Actually, the humidity during the preparation process significantly affected the formation of CPSMs, which was ascribed to the excellent water absorption property and longer evaporation speed of DMF. Moderate humidity environment (higher than $20 \% \mathrm{RH}$ and lower than $70 \%$ ) is more beneficial for CPSMs generation than low humidity $(<20 \% \mathrm{RH})$. Therefore, when placed in a drying oven, there were no CPSMs but film obtained (Fig. S8 $\dagger$ ). Besides, we also prepared PPFOH spincoated film from DMF solution. As shown in the SEM images of the film (Fig. S9†), inhomogeneous and aggregated CPSMs but smaller sizes formed on the continuous film. In this regard, DMF evaporation rate is an important factor to dominate the formation of $\mathrm{PPFOH}$-based CPSMs, that rapid evaporation is unfavorable for the formation of spheres. Here, we propose a possible formation process: first, $\mathrm{PPFOH}$ is dissolved in DMF solution due to the formation of hydrogen bonds between hydroxyl groups linked to the polymer backbones and carbonyl groups on the DMF molecule. With the DMF evaporation, DMFhydrophobic backbone chains fold and then form spheres, which are surrounded by the hydrophilic hydrogen bonds outside effectively. The absorption of water molecules can also disrupt hydrogen-bonds between $\mathrm{PPFOH}$ and DMF, further promoted the formation of spheres. During solvent evaporation process, PPFOH eventually assembles into well-defined CPSMs.
Our results suggested that supramolecular functionalization can afford semi-rigid conjugated polymer with amphiphilic property in a certain solvent and give rise to self-assemble into size-controllable CPSMs.

The close packing of PPFOH CPSMs can be a model to construct fluorescent colloidal crystals after fine manipulation (Fig. 1c and d). We tried to create three-dimensional (3D) colloidal crystals of CPSMs preliminarily. According to the formation mechanism of CPSMs, we further designed a simple droplet-pinned solvent evaporation method (Fig. 1j). ${ }^{34}$ The operating principle of this approach is illustrated as follows: during drying of a droplet of the polymer solution on a substrate, CPSMs nucleate near the contact line and grow gradually along the receding direction (toward the centre) of the droplet, ultimately closely stack together. Large-scale $\mathrm{PPFOH}$ CPSMs film (up to $100 \mu \mathrm{m}$ ) with regular arrangement was obtained by this method (Fig. 1f and g). PPFOH CPSMs in the large-scale film show the similar diameter and smooth surface with a size about $600 \mathrm{~nm}$ (Fig. $1 \mathrm{~h}$ and i). ${ }^{11}$ Certainly, more attempts are needed to create colloidal crystals, such as precisely controlling of the size and deviation. Here, we provide a significant potential candidate for photonic crystals using supramolecular functionalization of $\pi$-conjugated polymers.

The optical properties of PPFOH CPSMs and solutions were investigated by the UV-vis absorption and photoluminescence (PL) measurements (Fig. 2). It is easy to distinguish that the fluorescent profiles of PPFOH CPSMs are composed of two emission regimes: the blue emission at 440-460 $\mathrm{nm}$ and the green-yellow emission at $540-560 \mathrm{~nm}$, which is different from the pristine PFO nanoparticles prepared via re-precipitation with the absence of green-yellow band emission, ${ }^{35}$ but similar to the amphiphilic oligofluorene- or polyfluorene-based nanoparticles (also confirmed a slightly amphiphilic property of PPFOH@DMF complex in DMF solution). ${ }^{36-38}$ Interestingly, the 
(a)

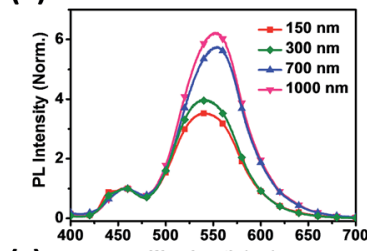

(c)

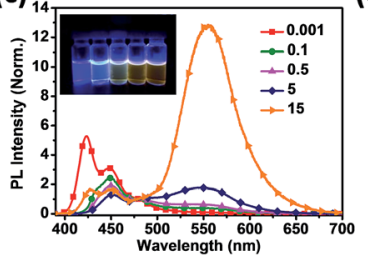

(b)

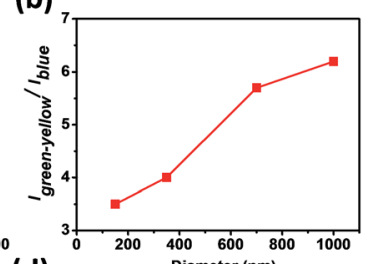

(d)

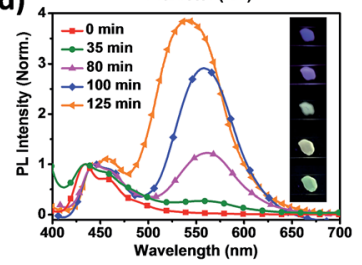

Fig. 2 (a) Photoluminescence (PL) spectra of PPFOH CPSMs with different diameters. (b) Green-yellow index of PPFOH with the function of the CPSMs diameter. (c) Concentration dependent PL spectra of PPFOH@DMF solution, the inset showing the photograph of fluorescent dispersions of PPFOH in DMF under the UV lamp $(365 \mathrm{~nm}$ ) (left to right: $0.001 \mathrm{mg} \mathrm{mL}^{-1}$ to $15 \mathrm{mg} \mathrm{mL}^{-1}$ ). PL spectra of concentrated solutions are measured by using the front-face detection from a $1 \mathrm{~mm}$ cuvette to extremely minimize the effect of self-absorbance on $\mathrm{PL}$ spectra. (d) $\mathrm{PL}$ spectra of $1 \mathrm{mg} \mathrm{mL}^{-1}$ PPFOH/DMF solution taken at different drying time at $20{ }^{\circ} \mathrm{C}$ (without daylight), the inset showing the photographs of PPFOH/DMF solution dropped on the silicon substrate during the solvent evaporation process (top to down: 0 min to 125 min). All above were excited at $380 \mathrm{~nm}$.

low band emission of the larger CPSMs (>700 nm) red-shifted about $10 \mathrm{~nm}$ in comparison with those of smaller CPSMs $(<700 \mathrm{~nm})$. And the intensities of the broad and shoulder absorption peaks in the wavelength of $450-550 \mathrm{~nm}$ increased with the increasing CPSMs sizes (Fig. S10†). Two reasons may explain these properties: one is attributed to the size-dependent spectroscopic properties in closely stacked PPFOH CPSMs (lattice softening), ${ }^{39}$ the other is the stronger aggregation in the larger CPSMs. Time-resolved fluorescence spectroscopy measurements were carried out to determine the transition property of PPFOH CPSMs film, and the corresponding lifetimes were summarized in Table S1. $\uparrow$ PPFOH in DMF solution exhibited single-exponential $\left({ }^{1} \mathrm{~A}^{*}\right)$ decay at three detection wavelengths of 420,440 and $480 \mathrm{~nm}$, respectively, and their lifetimes were about $480 \mathrm{ps}$ corresponding to three peaks of the polyfluorenes fluorescent spectra ${ }^{40}$ But the energy transfer was significantly enhanced in the PPFOH CPSMs. It is clear to find that the high energy band emission $(420-480 \mathrm{~nm})$ disappeared or reduced sharply because of an increasing possibility of nonirradiative decay pathway for energy transfer from $450 \mathrm{~nm}$ (host, donor wavelength) to $560 \mathrm{~nm}$ (guest, acceptor wavelength). The lifetime analysis was implemented at 450 and $560 \mathrm{~nm}$, which were considered as donor and acceptor wavelengths, respectively (Fig. S10 and Table S1 $†$ ). The emission peak at $c a .560 \mathrm{~nm}$ exhibited double-exponential decay, and the lifetimes were about 2-4 ns and 6-9 ns, much longer than the blue emission at $450 \mathrm{~nm}$. It can be deduced that the aggregate species (low energy emission), together with the pristine polyfluorene chains in CPSMs (high energy emission), formed a self-doping nanosystem with energy transfer from host to guest. Meanwhile,

we also believed that this green band ( $g$-band) emission may be attributed to the aggregation excimer. Of course, our PPFOH also cannot completely avoid the oxidation in the preparation and purification processing, result in fluorenone-induced green emission. Also, our recent work indicated that the green emission may be ascribed to the random, distorted and folded fluorene conformation and/or therefore truncation of the conjugation length as the result of strong aggregation in CPSMs. ${ }^{41}$

The size-dependent PL spectra of CPSMs are shown in Fig. 2a. We clearly observed that the green-yellow index of CPSMs increased with the increasing of diameters (Fig. 2b). The possible reason is that the aggregate exciton is sensitive to the diameters of CPSMs owing to their comparable sizes, leading to a more effective energy transfer between single polymer chain (donor) and aggregates (acceptor). Noted that the stronger selfabsorption in the large size CPSMs film can also explain the size-dependent emission. Besides, the size-dependent $\mathrm{g}$-band emission is nearly linear proportional to the increased concentrations of $\mathrm{PPFOH} / \mathrm{DMF}$ solutions (Fig. 2c), which implies that the g-band emission is closely associated with the intensity of molecular aggregates. Compared with our previous work, where PPFOH nanoparticle formed immediately (kinetic processing), ${ }^{31}$ PPFOH CPSMs prepared by SEIS method undergo an extended period of time (thermodynamic processing) that resulted in the possible formation of aligned and stretched polymer chains and further induced excimer emission in the CPSMs (Fig. 2d). ${ }^{22}$ The emission peaks in the wavelength range of 540-560 nm increased with the increasing of drying time during the evaporation process. Therefore, molecular aggregation of $\pi$-stacked fluorene segments in spheres is an alternative explanation for the origin of the g-band emission. Even if consideration of fluorenone-mechanism, more efficient intraor interchain energy transfer can also explain the sizedependent emission.

We finally fabricated PPFOH spheres-based polymer nanostructure-based light-emitting devices (PNLEDs) to examine their semiconducting properties. Devices with the configuration of $\mathrm{Au} / \mathrm{p}-\mathrm{SiC} / \mathrm{PPFOH}$ spheres/ITO were fabricated to investigate the performance of CPSMs. ${ }^{42} \mathrm{CPSMs}$ films on the p-SiC (4H, Cree Inc.) can be easily prepared by drop-coating technique (Fig. 3a). Noted that larger PPFOH spheres with several hundred nanometers may stack together and further result into larger film thickness. In that case, no light emission could be observed from the PNLEDs. In general, PPFOH nanospheres with the diameters of $150 \mathrm{~nm}$ may tend to efficiently disperse on the p-SiC substrates. The average PPFOH-CPSMs film thickness in the PNLEDs is about $150 \mathrm{~nm}$. Fig. $3 \mathrm{~b}$ shows the current-voltage $(I-V)$ curve of the PNLEDs and the schematic diagram of the proposed PNLEDs configuration. Hence, the $I-V$ curve exhibited typical rectifying behaviour. The PNLEDs based on the CPSMs have turn-on voltage (current) of $\sim 25 \mathrm{~V}(0.60 \mathrm{~mA})$. The EL spectra of PNLEDs at various forward biased voltages are shown in Fig. $3 \mathrm{c}$ and no light emission was observed from the PNLEDs under reverse bias. The photograph of the devices indicated the white light emission (Fig. 3c, inset). The EL spectra of PNLEDs can be fitted by two Gaussian curves with 
(a)

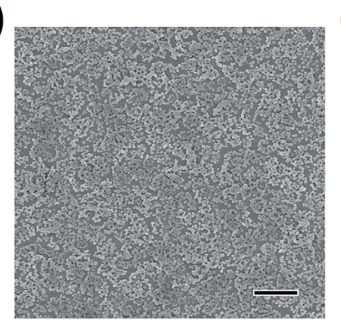

(c)

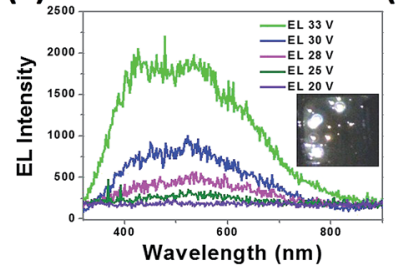

(b)

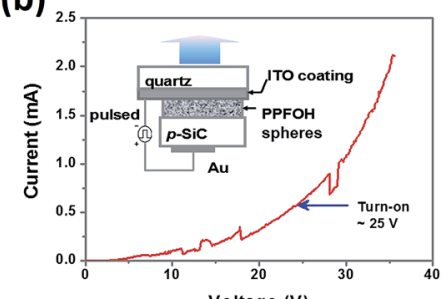

(d)

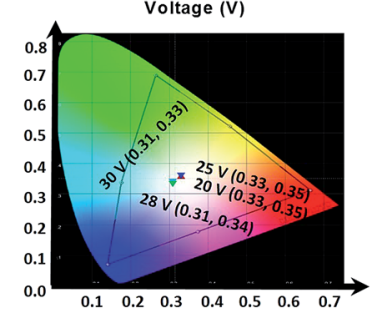

Fig. 3 (a) SEM images of the PPFOH-based films on the p-SiC, scale bar is $5 \mu \mathrm{m}$. (b) Current-voltage $(I-V)$ curve of the PNLEDs; the inset is the device configuration of the PNLEDs based on PPFOH spheres. (c) EL spectra at different voltages; the inset is the photograph of device with white light emission. (d) CIE coordinates of PNLEDs biased at various voltages.

peak wavelengths at 440 and $540 \mathrm{~nm}$, respectively. White light emission (CIE: $0.33,0.35$ ) can be observed by naked eyes from the surface of the PNLEDs in dark room (Fig. $3 \mathrm{c}$ and d). Notably, EL spectra showed a broad emission as compared with the PL spectra owing to the decreased green-yellow index. The EL intensity of the PNLEDs increased sharply when the applied voltages were larger than the turn-on voltage. In addition, no light emission under either optical or electrical excitation can be measured for the p-SiC sample without the PPFOH nanostructure layer in our experiments, hence the EL spectrum observed was due to the presence of PPFOH CPSMs.

\section{Conclusions}

In summary, we proposed the supramolecular functionalization strategy to design versatile hairy-rod type $\pi$-conjugated polymer amphiphiles that act as the precursor of supramolecular selfassembly for CPSMs. PPFOH CPSMs with different sizes have been obtained by controlling the initial concentration via SEIS method, which is a new and convenient method to prepare CPSMs thin-film on substrates or electrodes. The fascinating size-dependent luminescent properties of PPFOH CPSMs provide a flexible method to tune their emission colours. White light emission with CIE coordinates of $(0.33,0.35)$ has been achieved by single-component polyfluorene spheres in the PNLEDs. The closely packing of CPSMs would facilitate the construction of colloidal crystals. Supramolecular $\pi$-conjugated polymers are a series of potential next-generation advanced materials for organic optoelectronics.

\section{Acknowledgements}

We sincerely thank Professor Donal D. C. Bradley (University of Oxford) for valuable discussions. The project was supported by

the National Key Basic Research Program of China (973) (2015CB932200), National Natural Science Foundation of China (21504041, 21502091, 21274064, 61136003), Doctoral Fund of Ministry of Education of China (20133223110007), Project funded by the Priority Academic Program Development of Jiangsu Higher Education Institutions, PAPD (YX03001), Synergetic Innovation Center for Organic Electronics and Information Displays, China Postdoctoral Science Foundation (2015M580419), Jiangsu Planned Projects for Postdoctoral Research Funds (1501019B), the Six Peak Talents Foundation of Jiangsu Province (XCL-CXTD-009), Excellent Science and Technology Innovation Team of Jiangsu Higher Education Institutions (2013), Natural Science of the Education Committee of Jiangsu Province (15KJB430019). Dr Jinyi Lin gratefully acknowledges SICAM Fellowship, Oxford University, the Plastic Electronics Centre for Doctoral Training, UK EPSRC (EP/ G037515/1) and Imperial College London.

\section{Notes and references}

1 J. Burroughes, D. Bradley, A. Brown, R. Marks, K. Mackay, R. Friend, P. Burns and A. Holmes, Nature, 1990, 347, 539-541.

2 M. Campoy-Quiles, T. Ferenczi, T. Agostinelli, P. G. Etchegoin, Y. Kim, T. D. Anthopoulos, P. N. Stavrinou, D. D. Bradley and J. Nelson, Nat. Mater., 2008, 7, 158-164.

3 L.-H. Xie, C.-R. Yin, W.-Y. Lai, Q.-L. Fan and W. Huang, Prog. Polym. Sci., 2012, 37, 1192-1264.

4 W.-L. Yu, H. Meng, J. Pei and W. Huang, J. Am. Chem. Soc., 1998, 120, 11808-11809.

5 W. Huang, H. Meng, W. L. Yu, J. Gao and A. J. Heeger, Adv. Mater., 1998, 10, 593-596.

6 L.-H. Xie, S.-H. Yang, J.-Y. Lin, M.-D. Yi and W. Huang, Philos. Trans. R. Soc., A, 2013, 371, 20120337.

7 F. S. Kim, G. Ren and S. A. Jenekhe, Chem. Mater., 2010, 23, 682-732.

8 E. Fisslthaler, S. Sax, U. Scherf, G. Mauthner, E. Moderegger, K. Landfester and E. J. List, Appl. Phys. Lett., 2008, 92, 183305.

9 E. Fisslthaler, A. Blümel, K. Landfester, U. Scherf and E. J. List, Soft Matter, 2008, 4, 2448-2453.

10 T. Adachi, L. Tong, J. Kuwabara, T. Kanbara, A. Saeki, S. Seki and Y. Yamamoto, J. Am. Chem. Soc., 2013, 135, 870-876.

11 A. J. Kuehne, M. C. Gather and J. Sprakel, Nat. Commun., 2012, 3, 1088.

12 T. Kanai, T. Sawada, A. Toyotama and K. Kitamura, Adv. Funct. Mater., 2005, 15, 25-29.

13 A. Molinos-Gómez, M. Maymó, X. Vidal, D. Velasco, J. Martorell and F. López-Calahorra, Adv. Mater., 2007, 19, 3814-3818.

14 S. Furumi, T. Kanai and T. Sawada, Adv. Mater., 2011, 23, 3815-3820.

15 S. Satoh, H. Kajii, Y. Kawagishi, A. Fujii, M. Ozaki and K. Yoshino, Jpn. J. Appl. Phys., 1999, 38, L1475.

16 R. Polson, A. Chipouline and Z. V. Vardeny, Adv. Mater., 2001, 13, 760-764.

17 M. Deutsch, Y. A. Vlasov and D. J. Norris, Adv. Mater., 2000, 12, 1176-1180. 
18 H. Yabu, A. Tajima, T. Higuchi and M. Shimomura, Chem. Commun., 2008, 4588-4589.

19 S. John and T. Quang, Phys. Rev. Lett., 1997, 78, 1888.

20 K. Yoshino, S. Lee, S. Tatsuhara, Y. Kawagishi, M. Ozaki and A. Zakhidov, Appl. Phys. Lett., 1998, 73, 3506-3508.

21 J. P. Rao and K. E. Geckeler, Prog. Polym. Sci., 2011, 36, 887913.

22 J. Pecher and S. Mecking, Chem. Rev., 2010, 110, 6260-6279.

23 D. Tuncel and H. V. Demir, Nanoscale, 2010, 2, 484-494.

24 P. M. Beaujuge and J. M. Fréchet, J. Am. Chem. Soc., 2011, 133, 20009-20029.

25 X. L. Chen and S. A. Jenekhe, Macromolecules, 2000, 33, 4610-4612.

26 H. Sirringhaus, P. Brown, R. Friend, M. M. Nielsen, K. Bechgaard, B. Langeveld-Voss, A. Spiering, R. A. Janssen, E. Meijer and P. Herwig, Nature, 1999, 401, 685-688.

27 X. Xiao, W. Bai, L. Cai and J. Lin, Chem. Lett., 2014, 43, 331333.

28 X. Xiao, W. Bai and J. Lin, Polym. Bull., 2014, 71, 2103-2112.

29 F. J. Hoeben, P. Jonkheijm, E. Meijer and A. P. Schenning, Chem. Rev., 2005, 105, 1491-1546.

30 R. C. Evans, J. Mater. Chem. C, 2013, 1, 4190-4200.

31 J. Y. Lin, J. Wong, L. H. Xie, X. C. Dong, H. Y. Yang and W. Huang, Macromol. Rapid Commun., 2014, 35, 895-900.
32 D. M. Bassani, L. Jonusauskaite, A. Lavie-Cambot, N. D. McClenaghan, J.-L. Pozzo, D. Ray and G. Vives, Coord. Chem. Rev., 2010, 254, 2429-2445.

33 J. Lin, Z. Yu, W. Zhu, G. Xing, Z. Lin, S. Yang, L. Xie, C. Niu and W. Huang, Polym. Chem., 2013, 4, 477-483.

34 H. Li, B. C. Tee, J. J. Cha, Y. Cui, J. W. Chung, S. Y. Lee and Z. Bao, J. Am. Chem. Soc., 2012, 134, 2760-2765.

35 C. Wu and J. McNeill, Langmuir, 2008, 24, 5855-5861.

36 L. Zhu, J. Qin and C. Yang, J. Phys. Chem. B, 2010, 114, 1488414889.

37 L. Zhu, C. Yang and J. Qin, Chem. Commun., 2008, 63036305.

38 F. Wang, M.-Y. Han, K. Y. Mya, Y. Wang and Y.-H. Lai, J. Am. Chem. Soc., 2005, 127, 10350-10355.

39 N. Kurokawa, H. Yoshikawa, N. Hirota, K. Hyodo and H. Masuhara, ChemPhysChem, 2004, 5, 1609-1615.

40 J.-Y. Lin, W.-S. Zhu, F. Liu, L.-H. Xie, L. Zhang, R. Xia, G.-C. Xing and W. Huang, Macromolecules, 2014, 47, 10011007.

41 Y.-Y. Liu, J.-Y. Lin, Y.-F. Bo, L.-H. Xie, M.-D. Yi, X.-W. Zhang, H.-M. Zhang, T.-P. Loh and W. Huang, Org. Lett., 2015, 18, 172-175.

42 J. Xiao, H. Yang, Z. Yin, J. Guo, F. Boey, H. Zhang and Q. Zhang, J. Mater. Chem., 2011, 21, 1423-1427. 